\title{
Erratum
}

\section{The $N \bar{N}$ and $\pi \pi$ Decay Modes of Baryonium}

\author{
I. M. Barbour and J. P. Gilchrist
}

Z. Phys. C - Particles and Fields 7, 225 (1981)

The factor $(2 x+1)^{-1}$ in (7) is incorrect and should be replaced by $[(2 x+1) !]^{-1}$. The predicted widths to $N \bar{N}$ for the Baryonium states shown in Table 2 are consequently incorrect. States with widths to $N \bar{N}>40 \mathrm{MeV}$ are summarized below

\begin{tabular}{llll}
\hline Mass $(\mathrm{GeV})$ & $J^{P}$ & $I^{G}$ & $N \bar{N}$ Widths $(\mathrm{MeV})$ \\
\hline 2.02 & $0^{-}$ & $0^{+}$ & 66 \\
2.02 & $0^{-}$ & $1^{-}$ & 44 \\
2.07 & $1^{-}$ & $0^{-}$ & 52 \\
1.97 & $2^{+}$ & $0^{+}$ & 139 \\
2.14 & $1^{+}$ & $1^{+}$ & 66 \\
214 & $2^{+}$ & $1^{-}$ & 80 \\
2.32 & $4^{+}$ & $0^{+}$ & 54 \\
2.21 & $3^{-}$ & $0^{-}$ & 147 \\
2.37 & $3^{-}$ & $1^{+}$ & 53 \\
2.37 & $4^{-}$ & $1^{+}$ & 44 \\
2.54 & $5^{-}$ & $0^{-}$ & 52 \\
\hline
\end{tabular}

These results still suggest that typical widths of $T$-baryonium are of the order of $100 \mathrm{MeV}$. The other conclusions of the paper are unaltered.

Acknowledgements. We would like to thank J. P. Ader and S. Sood for pointing out the error in (7) and for a useful correspondence. 